\title{
Phaseless Microwave Tomography Assessment for Breast Imaging: Preliminary Results
}

\author{
Sandra Costanzo $\mathbb{i D}^{1,2,3}$ and Giuseppe Lopez ${ }^{1}$ \\ ${ }^{1}$ DIMES, University of Calabria, 87036 Rende (CS), Italy \\ ${ }^{2}$ ICEmB, Inter-University National Research Center on Interactions between Electromagnetic Fields and Biosystems, \\ 16145 Genova, Italy \\ ${ }^{3}$ CNR-Institute for Electromagnetic Sensing of the Environment (IREA), Via Diocleziano 328, 80124 Naples, Italy \\ Correspondence should be addressed to Sandra Costanzo; costanzo@dimes.unical.it
}

Received 19 July 2019; Accepted 23 January 2020; Published 22 February 2020

Academic Editor: Ping Li

Copyright (c) 2020 Sandra Costanzo and Giuseppe Lopez. This is an open access article distributed under the Creative Commons Attribution License, which permits unrestricted use, distribution, and reproduction in any medium, provided the original work is properly cited.

In the present work, a phaseless approach for microwave imaging applications is presented. The proposed solution strategy is based on the formulation of the scattering phenomena in terms of contrast source, while no phase-recovery stage is involved into the numerical procedure, thus providing a phaseless single-step resolution method. The image recovering potentialities of the discussed method are numerically validated by successfully distinguishing different tissues of a slice breast model, with a tumor located wherein. The above preliminary assessment encourages the adoption of the proposed solution in the framework of biomedical imaging.

\section{Introduction}

Recent trends in biomedical imaging have raised some concerns about the safety of the imaging apparatus, as well as about the overall system costs. Microwave tomography (MWT) can be regarded as a safe, cost-effective, noninvasive supplement to the widely adopted imaging techniques. However, limited penetration depth and relatively low resolution put some constraints on a large-scale application of MWT. A major reason to move towards the microwave spectrum is given by the possibility to identify possible pathological alterations on biological tissues according to their water content. Furthermore, the use of nonionizing radiation in MWT techniques represents one of the main advantages as compared to computed tomography (CT) and positron emission tomography (PET). In this work, according to the preliminary assessment presented in [1], a Phaseless Contrast Source Inversion (P-CSI) method is successfully implemented for breast tissue reconstruction. Specifically, starting from the contrast source (CS) formulation described in $[2,3]$, the inverse scattering problem is solved with no linearization procedure, but recasting it into an iterative optimization problem, where the two problems unknowns, namely, the contrast source and the dielectric contrast, are alternatively updated according to a conjugate gradient scheme. The inversion procedure is performed by exploiting the amplitude-only data of the measured total field, locally defined as the sum of the incident field and the scattered field, the former obtained as a base-line measurement in the absence of the object under test (OUT), and the latter due to the interaction of the incident field with the OUT. The full-data information of the incident field inside the imaging domain, obtained through a wave expansion starting from the base-line measurement, is also required into the reconstruction process.

\section{Phaseless Inverse Scattering Formulation}

Let us consider a $2 \mathrm{D}$ tomography problem, aiming at retrieving the shape, the location and the dielectric properties of a generic OUT, hereby denoted as B. A TM-polarized incident field is assumed, and cylindrical targets are investigated. 
In the whole scenario, a magnetic permittivity equal to that of free space is assumed, such as in a typical biomedical scenario, due to the nonmagnetic properties of the biological tissues. Therefore, the electromagnetic properties are fully described uniquely by the dielectric permittivity values. A circular acquisition setup is considered, where the measurement points are displaced on the acquisition curve $S$, as shown in the general scheme of Figure 1. It describes a multistatic and multiview setup, where the transmitter location is alternatively changed, thus determining $\mathrm{N}_{\mathrm{TX}}$ angles of incidence and resulting into a number of $\mathrm{N}_{\mathrm{R}} \cdot \mathrm{N}_{\mathrm{TX}}$ measurements. The imaging domain, hereby indicated as $D$, fully contains the unknown OUT.

The inverse scattering problem is governed by the electrical field integral equations (EFIEs), known in the literature as data equation and state equation, and obtained as solution of the Helmholtz equation applied to the aforementioned setup [4]:

$$
\begin{aligned}
& E^{s}(r)=E^{t}(r)-E^{i}(r)=k_{b}^{2} \int_{D} G\left(r, r^{\prime}\right) \chi\left(r^{\prime}\right) E^{t}\left(r^{\prime}\right) \mathrm{d} r^{\prime}, \quad r \in S, \\
& E^{t}(r)=E^{i}(r)+k_{b}^{2} \int_{D} G\left(r, r^{\prime}\right) \chi\left(r^{\prime}\right) E^{t}\left(r^{\prime}\right) \mathrm{d} r^{\prime}, \quad r \in D .
\end{aligned}
$$

In the above expressions, $E^{s}$ represents the scattered field, while $E^{t}, E^{i}$ give the total and the incident field, respectively; $G$ denotes the Green's function of the background medium, while parameter $\chi$ gives the contrast function, normalized with respect to the permittivity of the background medium:

$$
\chi(r)= \begin{cases}\frac{k^{2}(r)}{k_{b}^{2}}-1, & r \in B, \\ 0, & r \notin B .\end{cases}
$$

The considered approach is mathematically arranged into a local optimization problem, wherein a proper cost function is to be defined. The solution of the scattering problem is obtained as an iterative interplay of the EFIEs expressed by equations (1) and (2), which is formulated by the following cost function:

$$
F\left(\omega_{v, n}\right):=F_{S}\left(\omega_{v, n}\right)+F_{D}\left(\omega_{v, n}, \chi_{n}\right)
$$

with

$$
\begin{gathered}
F_{S}\left(\omega_{v, n}\right)=\alpha_{\mathrm{S}} \sum_{v}\left\|\left|f_{v}\right|^{2}-\left|E_{v}^{i}+G_{S} \omega_{v}\right|^{2}\right\|_{S}^{2} \\
F_{D}\left(\omega_{v, n}, \chi_{n}\right)=\alpha_{\mathrm{D}} \sum_{v}\left\|\chi\left[E_{v}^{i}+G_{D} \omega_{v}\right]-\omega_{v}\right\|_{D}^{2}
\end{gathered}
$$

In the above expressions, parameters $\alpha_{\mathrm{S}-\mathrm{D}}$ denote proper normalization factors. The expressions shown in equation (5) represent the discrepancy between the measured total field in $\mathrm{S}$, indicated as $f$, and the relative value according to the model defined into equation (1), while equation (6) considers the error affected by equation (2). Therefore, the cost function $F$ combines the cumulative difference between

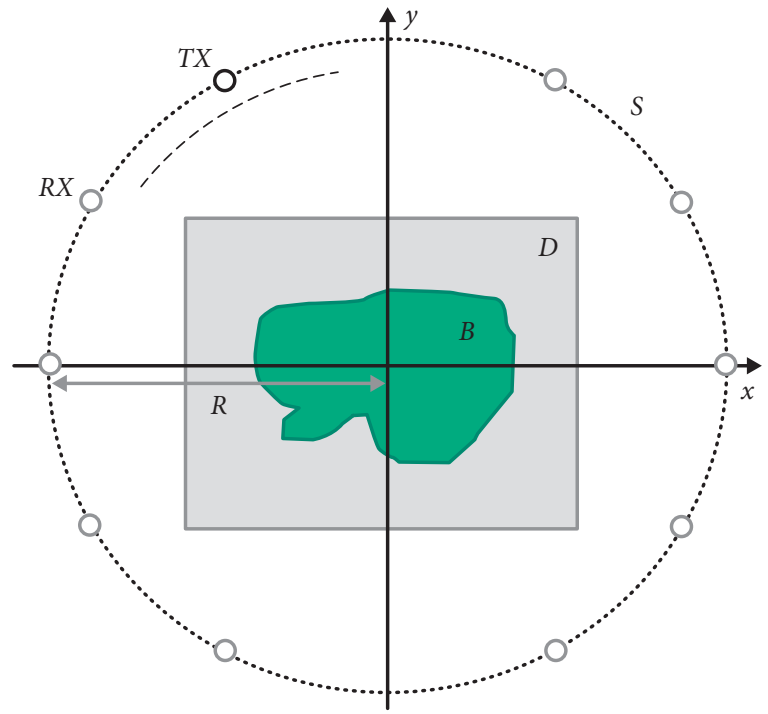

FIgURe 1: Acquisition setup for imaging.

the analytical data and the measured field for every illumination scenario $v$. The incident field in the OUT is determined starting from the incident electric field measurements performed along the acquisition curve $S$, by expressing the incident field as the sum of a limited number $\mathrm{N}$ of Hankel functions of the second kind $[5,6]$, so that the following relation holds true:

$$
E_{S}^{i}(\rho, \theta) \cong-\frac{j}{4} \sum_{\nu=-N}^{N} c_{\nu} H_{\nu}^{(2)}\left(k_{b} \rho\right) \exp (j \nu \theta),
$$

where $\rho$ and $\vartheta$ represent the polar coordinates.

The truncation number $N$ is determined by evaluating the variation of the Hankel function with respect to $\mathrm{N}$, for a fixed parameter $\rho$ equal to the radius $R$ of the sampling curve, as shown in Figure 2. The computed $c_{\nu}$ coefficients are subsequently implemented in the evaluation of the incident field inside the OUT [7-9].

The iterative process starts by updating the contrast source $\omega_{v, n}$, through the adoption of a nonlinear conjugate gradient scheme $[10,11]$, while the contrast function $\chi_{n}$, after the evaluation of the current value of the total field at the n-th step, $E_{v, n}^{t}=E_{v}^{i}+G_{S} \omega_{v, n}$, is obtained as a minimizer of the state function, indicated as $F_{D}\left(E_{v, n}^{t}, \omega_{v, n}\right)$. Since an iterative local-based optimization problem is involved, an initial guess for the contrast source $\omega_{v, 0}$ occurs. Therefore, a proper initial value for the contrast source is considered, able to exploit all the available information provided by the measurement step. The initial guess is obtained by applying the steepest descent method to equation (5), thus resulting into the following expression:

$$
\omega_{v, 0}=-2 \beta \alpha_{S} G_{S}^{*}\left[E_{v}^{i}\left(\left|f_{v}\right|^{2}-\left|E_{v}^{i}\right|^{2}\right)\right] F_{S},
$$

where $\beta$ is the step size, while the symbol $(*)$ indicates the adjoint operator. According to equation (8), all the available data are fully exploited in the initialization of the contrast 


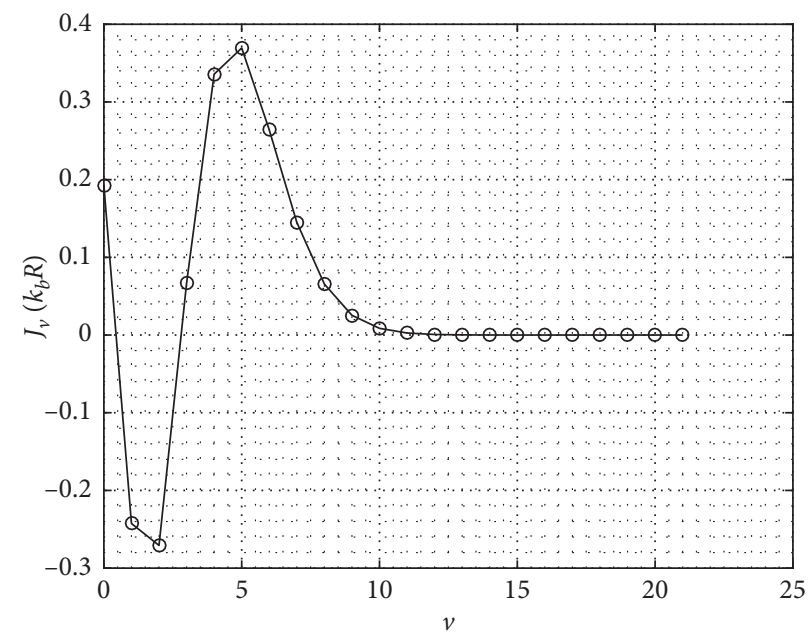

FIgURE 2: Bessel function behaviour versus the order $v$.

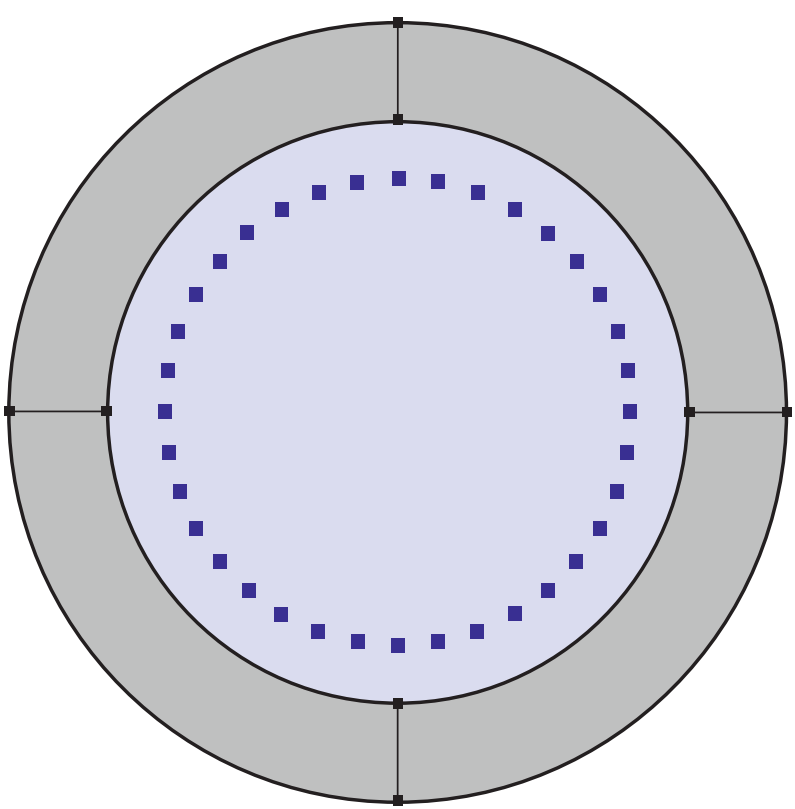

Figure 3: Acquisition setup on COMSOL: PML (grey), TX antenna positions (blue),. and matching medium (light blue).

source, with no arbitrary assumption on the unknown phase of the total field, which is instead proposed in $[10,11]$. Furthermore, no a priori information about the dielectric contrast inside $D$ is required, as compared to the approach outlined in [12].

\section{Human Breast Modelling and Imaging Results}

To perform a numerical validation of the proposed inverse strategy, a proper microwave imaging setup is simulated on COMSOL Multiphysics ${ }^{\circledR}$ [13], according to the proposed reconstruction method. The simulated setup is shown in Figure 3. It consists of a multistatic measurement setup, where $\mathrm{N}_{\mathrm{TX}}$ point current source is uniformly arranged in a circular fashion, by surrounding the OUT. The $2-\mathrm{GHz}$ operating frequency is considered, which is the most suitable one for microwave breast imaging, as it offers a considerable trade-off between spatial resolution and penetration depth into human breast tissues [14]. A perfectly matched layer (PML) surrounding the background medium is assumed as boundary condition. The forward data computation is performed by alternatively evaluating the total field and the incident field for a fixed position of the transmitting antenna. In order to speed up the simulation process, out-ofplane line currents are implemented as TM sources.

The dielectric profile of the breast is constructed similarly to the approach outlined in [14]. In particular, a simplified 3-tissue breast model is considered, whose dielectric properties are listed in Table 1. The implemented human breast model consists of a cylinder with a $45.5 \mathrm{~mm}$ radius, a fibroglandular region having a radius equal to 
TABle 1: Dielectric properties of breast tissues at $2 \mathrm{GHz}[14,15]$.

\begin{tabular}{lcc}
\hline Tissue & Relative permittivity & Conductivity $(\mathrm{S} / \mathrm{m})$ \\
\hline Fat & 5 & $5.0 E-02$ \\
Glandular/fibroconnective tissue & 43 & 0.7 \\
Malignant tissue & 35 & 0.7 \\
\hline
\end{tabular}

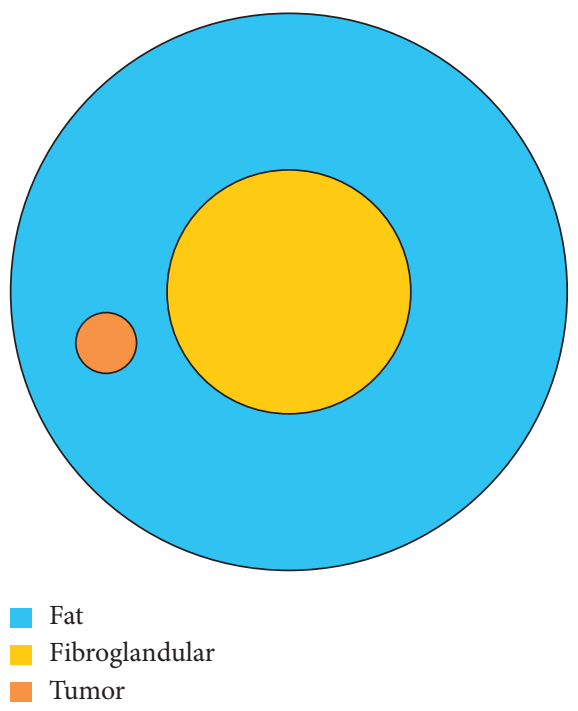

Figure 4: First scenario: tumor located inside the fat tissue.

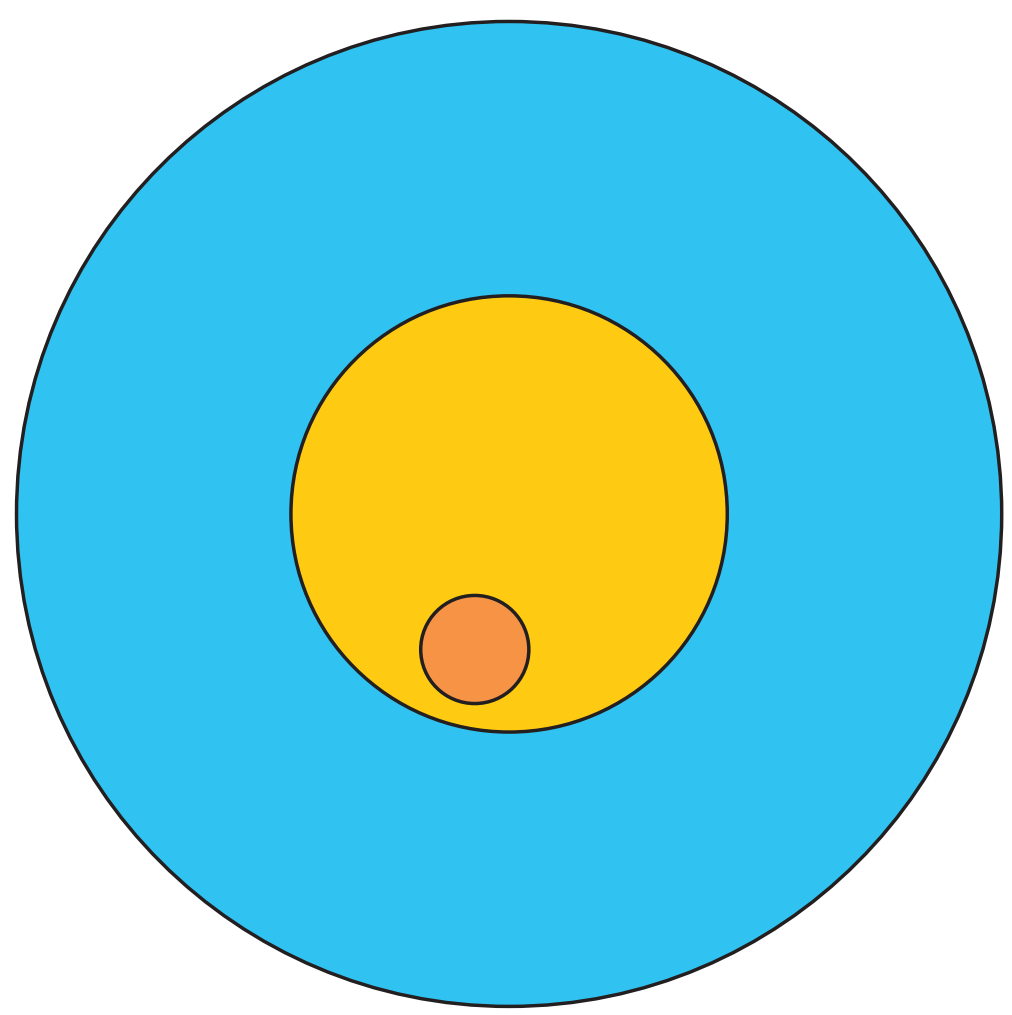

FIGURE 5: Second scenario: tumor located inside the fibroglandular tissue.

$20 \mathrm{~mm}$; the assumed tumor has a radius equal to $5 \mathrm{~mm}$. The probe and the transmitter locations are placed $15 \mathrm{~mm}$ far from the breast, in a circular fashion. A lossless matching medium with $\varepsilon_{\mathrm{r}}=12$ is assumed.

\section{Results and Discussion}

Firstly, the cancerous portion is placed inside the adipose tissue (Figure 4), where a higher dielectric contrast can 


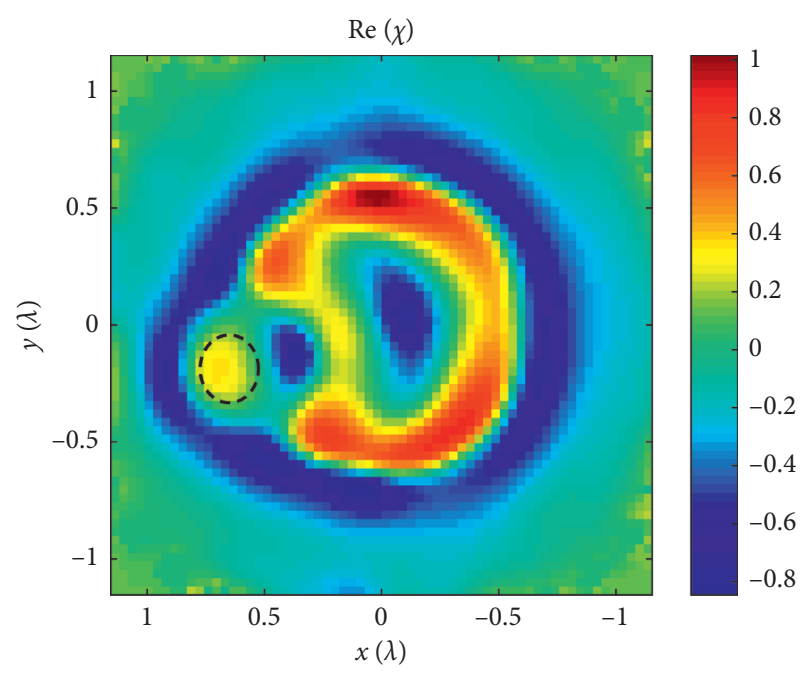

(a)

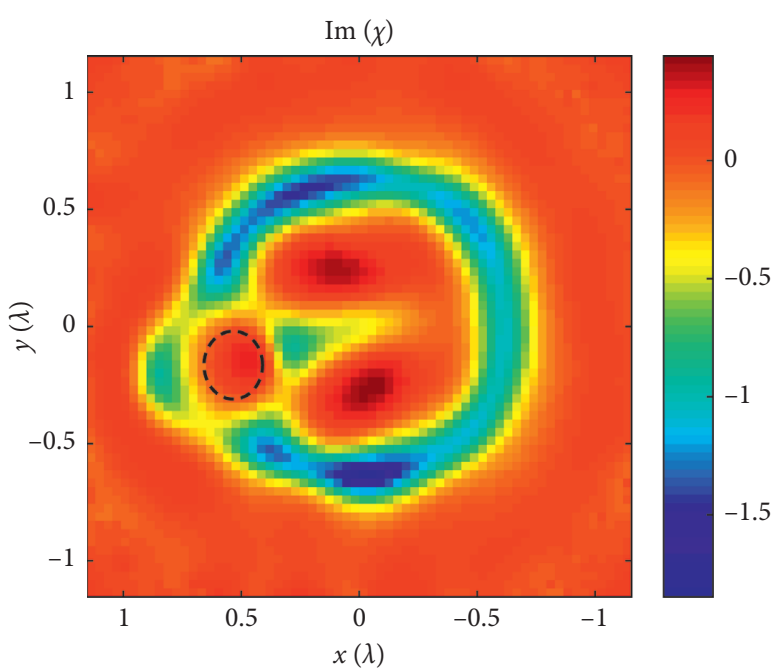

(b)

Figure 6: Real (a) and imaginary (b) part of the contrast function $\chi$ for the first scenario.

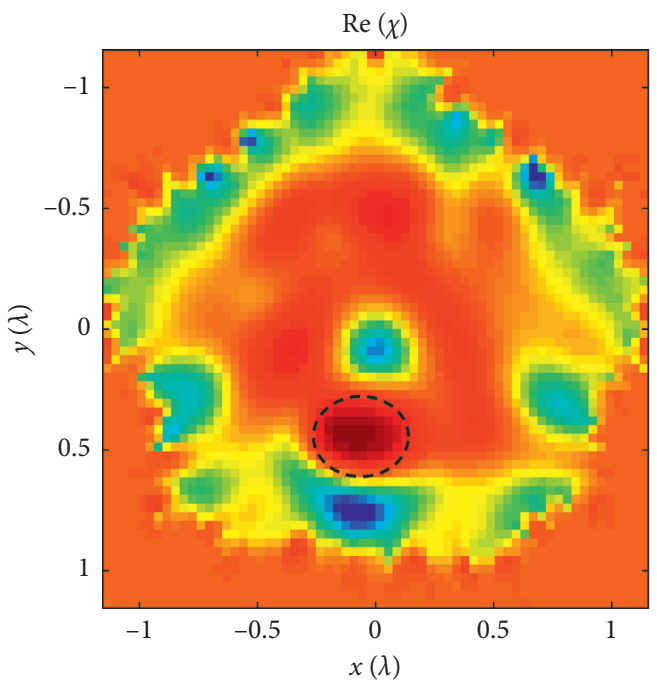

(a)

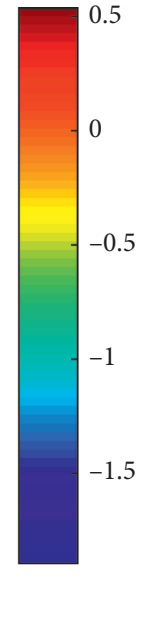

Figure 7: Real (a) and imaginary (b) part of the contrast function $\chi$ for the second scenario. be experienced. A second case is then considered, with the tumor located inside the fibroglandular tissue (Figure 5), thus resulting into a lower dielectric contrast. As clearly shown in the contrast function map of Figures 6 and 7, for the two scenarios, the tumor is properly localized in both cases.

\section{Conclusions}

A single-step phaseless microwave tomography method for biomedical imaging has been proposed in this work. Firstly, the analytical formulation of the inverse scattering procedure has been discussed, by introducing a novel initialization for the algorithm. Secondly, some reconstruction results of a slice breast model have been presented, where the reconstruction capabilities of the

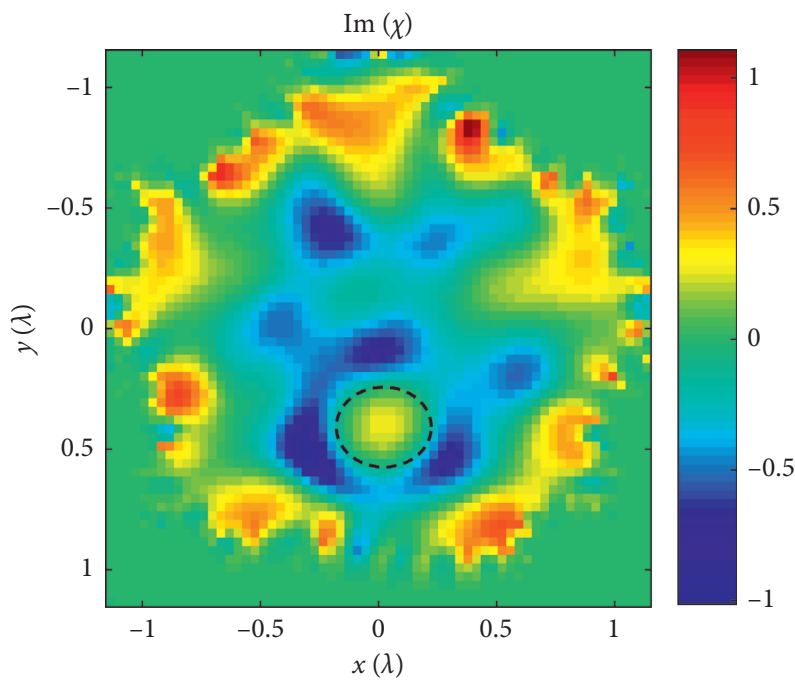

(b) proposed methodology have been confirmed. The discussed results represent a preliminary assessment for the use of MWT as a potential low-cost tool in the field of breast cancer detection and breast imaging applications. Further developments are currently being performed in order to achieve a quantitative reconstruction in terms of dielectric properties for the under test scenario.

\section{Data Availability}

The simulation data used to support the findings of this study are included within the article.

\section{Conflicts of Interest}

The authors declare that they have no conflicts of interest. 


\section{References}

[1] S. Costanzo and G. Lopez, Single-Step Approach to Phaseless Contrast-Source Inverse Scattering, Springer, Cham, Switzerland, 2019.

[2] P. M. Van Den Berg and A. Abubakar, "Contrast source inversion method: state of art-abstract," Journal of Electromagnetic Waves and Applications, vol. 15, no. 11, pp. 15031505, 2001.

[3] P. M. v. d. Berg and R. E. Kleinman, "A contrast source inversion method," Inverse Problems, vol. 13, no. 6, pp. 16071620, 1997.

[4] S. Costanzo, G. Di Massa, M. Pastorino, A. Randazzo, and A. Borgia, "Non-invasive microwave characterization of dielectric scatterers," in Microwave Materials Characterization, InTech, London, UK, 2012.

[5] D. Colton and R. Kress, "The Helmholtz equation," in Inverse Acoustic and Electromagnetic Scattering Theory, pp. 13-38, Springer, Berlin, Germany, 2013.

[6] T. Tsuburaya, Z. Meng, and T. Takenaka, "Inverse scattering analysis from measurement data of total electric and magnetic fields by means of cylindrical-wave expansion," Electronics, vol. 8, no. 4, p. 417, 2019.

[7] E. Mallikarjun and A. Bhattacharya, "Method to calculate the incident fields in the investigation domain in the perspective of permittivity reconstruction," IET Microwaves, Antennas \& Propagation, vol. 9, no. 3, pp. 252-263, 2015.

[8] F. Bürgel, K. S. Kazimierski, and A. Lechleiter, "A sparsity regularization and total variation based computational framework for the inverse medium problem in scattering," Journal of Computational Physics, vol. 339, pp. 1-30, Jun. 2017.

[9] F. Bürgel, K. S. Kazimierski, and A. Lechleiter, "IPscatt-a MATLAB Toolbox for the Inverse Medium Problem in Scattering," ACM Transactions on Mathematical Software (TOMS), vol. 45, no. 4, pp. 1-20, 2017.

[10] Z. Hu, L. Lianlin, and L. Fang, "A multi-frequency MRCSI algorithm with phaseless data," Inverse Probl, vol. 25, no. 6, Article ID 065006, 2009.

[11] L. Li, H. Zheng, and F. Li, "Two-dimensional contrast source inversion method with phaseless data: TM case," in IEEE Transactions on Geoscience and Remote Sensing, vol. 47, no. 6 , pp. 1719-1736, 2009.

[12] M. D’Urso, K. Belkebir, L. Crocco, T. Isernia, and A. Litman, "Phaseless imaging with experimental data: facts and challenges," Journal of the Optical Society of America, vol. 25, no. 1, p. 271, 2008.

[13] COMSOL AB, “COMSOL Multiphysics ${ }^{\circledR}$. v. 5.4, COMSOL AB, Stockholm, Sweden, 2012, http://www.comsol.com.

[14] G. Bellizzi, O. M. Bucci, and I. Catapano, "Microwave cancer imaging exploiting magnetic nanoparticles as contrast agent," IEEE Transactions on Biomedical Engineering, vol. 58, no. 9, pp. 2528-2536, 2011.

[15] "Dielectric properties of body tissues," 2019, http://niremf. ifac.cnr.it/tissprop/\#appl. 